\author{
G. Lattuada $\cdot$ F. Costantino $\cdot$ A. Caumo $\cdot$ P. Scifo • \\ F. Ragogna · F. De Cobelli • A. Del Maschio $\cdot$ L. Luzi • \\ G. Perseghin
}

\title{
Reduced whole-body lipid oxidation is associated with insulin resistance, but not with intramyocellular lipid content in offspring of type 2 diabetic patients
}

Received: 6 July 2004 / Accepted: 14 November 2004 / Published online: 10 March 2005

C) Springer-Verlag 2005

\begin{abstract}
Aims/hypothesis: Intramyocellular lipid accumulation and insulin resistance are thought to be due to reduced lipid oxidation in a human model of high risk of developing type 2 diabetes. Methods: We studied 32 offspring of type 2 diabetic parents and 32 control individuals
\end{abstract}

\author{
G. Lattuada - F. Costantino - A. Caumo - F. Ragogna \\ L. Luzi · G. Perseghin $(\bowtie)$ \\ Internal Medicine-Section of Nutrition/Metabolism, \\ Scientific Institute H San Raffaele, \\ via Olgettina 60 , \\ 20132 Milan, Italy \\ e-mail: perseghin.gianluca@hsr.it \\ Tel.: +39-02-26432614 \\ Fax: +39-02-26432771 \\ P. Scifo \\ Division of Nuclear Medicine, \\ Scientific Institute H San Raffaele, \\ via Olgettina 60 , \\ 20132 Milan, Italy \\ P. Scifo · F. De Cobelli - A. Del Maschio \\ Division of Diagnostic Radiology, \\ Scientific Institute H San Raffaele, \\ via Olgettina 60 \\ 20132 Milan, Italy \\ P. Scifo - F. De Cobelli - A. Del Maschio - L. Luzi • \\ G. Perseghin \\ Unit of Clinical Spectroscopy, \\ Scientific Institute H San Raffaele, \\ via Olgettina 60 , \\ 20132 Milan, Italy
}

\section{A. Del Maschio}

Università Vita e Salute San Raffaele,

Scientific Institute H San Raffaele,

via Olgettina 60 ,

20132 Milan, Italy

L. Luzi

Faculty of Exercise Sciences,

Università degli Studi di Milano,

Italy by means of DXA, indirect calorimetry, insulin clamp and ${ }^{1} \mathrm{H}$ MRS of the calf muscles, and differences between and within study groups were analysed before and after segregation by quartiles of fasting lipid oxidation. Results: In comparison with control subjects, the offspring showed impaired insulin sensitivity, which was associated with higher fasting intramyocellular lipid content (Spearman's rho $-0.35 ; p=0.04$ ), but fasting lipid oxidation did not differ between groups $\left(1.21 \pm 0.46\right.$ vs. $1.25 \pm 0.37 \mathrm{mg} \cdot \mathrm{kg}^{-1}$ lean body mass per min; $p=0.70$ ). Nevertheless, offspring in the lowest quartile of lipid oxidation had the most severe impairment of insulin sensitivity and a strong association was shown between lipid oxidation and insulin sensitivity within quartiles (Spearman's rho $0.47 ; p=0.01$ ); this was not observed within the control group (Spearman's rho 0.13; $p=0.47$ ). Intramyocellular lipid content was not significantly different within quartiles of lipid oxidation in either of the groups. Conclusions/interpretation: Insulin sensitivity improved across increasing quartiles of fasting lipid oxidation in the offspring group, but remained constant in the control group, supporting the hypothesis that impaired fat oxidation is a primary pathogenic factor of insulin resistance in people with a genetic background for type 2 diabetes. Despite their association with impaired insulin sensitivity, soleus and tibialis anterior intramyocellular lipid content remained constant across increasing quartiles of fasting lipid oxidation within both groups.

Keywords Insulin resistance $\cdot$ Intramyocellular lipid content - Lipid oxidation - Offspring of type 2 diabetic parents

Abbreviations DXA: Dual-energy X-ray absorptiometry - EGP: Endogenous glucose production ${ }^{1} \mathrm{H}$-MRS: ${ }^{1} \mathrm{H}$-magnetic resonance spectroscopy - IMCL: Intramyocellular lipid - LBM: Lean body mass - OFF: Offspring of type 2 diabetic parents - REE: Resting energy expenditure $\cdot$ ROS: Reactive oxygen species $\cdot \mathrm{SI}_{\mathrm{P}(\mathrm{clamp})}$ : Clamp-based measure of peripheral insulin sensitivity . $\alpha$-TNFR-2: $\alpha$-Tumour necrosis factor receptor-2 


\section{Introduction}

Increased intramyocellular lipid (IMCL) content as assessed using ${ }^{1} \mathrm{H}$-magnetic resonance spectroscopy $\left({ }^{1} \mathrm{H}-\mathrm{MRS}\right)$ has been associated with insulin resistance in humans, especially in non-obese individuals [1-3]. In parallel, impairment of muscle or plasma NEFA oxidation in obesity and type 2 diabetes have been reported by different groups [46]. The observation that in obese women [5] and type 2 diabetic patients [7] the impairment of fat oxidation was not reversed by considerable body weight reduction and that it was also detected in individuals with impaired glucose tolerance [8] would support the hypothesis of a primary genetic defect. An impairment of fasting lipid oxidation in association with insulin resistance and IMCL accumulation may also be seen as a secondary consequence of metabolic disturbances [9]. The reverse metabolic picture was described by our group in healthy humans in whom, despite a moderate degree of overweight, insulin sensitivity and normal IMCL content were preserved in association with higher fasting lipid oxidation [10]. Moreover using a longitudinal approach, it was shown that in obese individuals enhanced insulin sensitivity through physical activity was associated with increased fat oxidation [11, 12].

Based on this evidence, the present work explores the hypothesis that IMCL accumulation and insulin resistance might be associated with reduced fasting whole-body lipid oxidation in non-diabetic offspring of type 2 diabetic parents (OFF), a well-established human model of primary and genetically determined insulin resistance.

\section{Subjects, materials and methods}

Description of study subjects The offspring group was recruited at Scientific Institute H. San Raffaele, Milan, Italy. The main criteria for their inclusion in the study were the following: (1) both parents with type 2 diabetes $(n=25)$ or one parent and a first-degree ( $n=4$, brother or sister) or second-degree $(n=3$; grandparents) relative with type 2 diabetes; (2) age (24-45 years); (3) white race; (4) sedentary life style (habitual physical activity was assessed using a questionnaire [13]); (5) no history of hypertension, endocrine/metabolic disease, or cigarette smoking. We selected 32 OFF (20F/12M) who did not participate in heavy physical activity. They were compared with 32 healthy subjects (NOR), who were carefully matched for anthropomorphic features with the exception that they had no family history of diabetes and hypertension traced through their grandparents. The characteristics of the study groups are summarised in Table 1. Body weight was stable for at least 6 to 12 months. Because of its potential impact on insulin sensitivity [14], women recruited for the study had not taken oral steroidal contraception for at least 12 months. All subjects were in good health as assessed by medical history and physical examination. Informed consent was obtained from all subjects after explanation of the purposes, nature and potential risks of the study. The protocol was approved by the Ethical Committee of the Scientific Institute H. San Raffaele.
Table 1 Characteristics of study groups

Values are means $\pm \mathrm{SD}$. $p$ values are for independent $t$-tests

${ }^{a}$ The range of possible scores for the physical activity index is 3 to 15 ; the lowest value corresponds to the level of physical activity of a clerical worker who plays a light sport (energy expended is $<0.76 \mathrm{MJ} / \mathrm{h}$, e.g., bowling) and who participates in sedentary activities during leisure time. The highest value corresponds to the level of physical activity of a person who is very physically active at work (e.g., a construction worker), who plays heavy sports (energy expanded is at least 1.76 $\mathrm{MJ} / \mathrm{h}$, e.g., boxing, basketball, football or rugby) and who is very physically active during leisure time (e.g., walking $>1 \mathrm{~h} /$ day or biking $>45 \mathrm{~min} /$ day)

\begin{tabular}{|c|c|c|c|}
\hline & Offspring & Normal & $p$ value \\
\hline Subjects: number (women/men) & $32(20 / 12)$ & $32(20 / 12)$ & \\
\hline \multicolumn{4}{|l|}{ Anthropometric features } \\
\hline Age (years) & $28 \pm 7$ & $26 \pm 5$ & 0.22 \\
\hline BMI $\left(\mathrm{kg} / \mathrm{m}^{2}\right)$ & $24.2 \pm 6.3$ & $23.8 \pm 5.7$ & 0.78 \\
\hline Body fat $(\%)$ & $28.6 \pm 12.0$ & $28.2 \pm 11.0$ & 0.89 \\
\hline LBM (kg) & $46 \pm 9$ & $47 \pm 10$ & 0.73 \\
\hline Physical activity index ${ }^{a}$ & $9.0 \pm 0.3$ & $9.0 \pm 0.5$ & 0.94 \\
\hline \multicolumn{4}{|l|}{ Laboratory features } \\
\hline Plasma glucose (mmol/l) & $5.2 \pm 0.8$ & $5.0 \pm 0.3$ & 0.11 \\
\hline Plasma total cholesterol $(\mathrm{mmol} / \mathrm{l})$ & $4.91 \pm 0.80$ & $4.42 \pm 0.64$ & 0.02 \\
\hline Plasma triglyceride $(\mathrm{mmol} / \mathrm{l})$ & $1.07 \pm 0.44$ & $0.84 \pm 0.34$ & 0.12 \\
\hline Plasma NEFA (mmol/l) & $0.642 \pm 0.183$ & $0.481 \pm 0.208$ & $<0.01$ \\
\hline \multicolumn{4}{|l|}{ Energy expenditure } \\
\hline REE (kJ/day) & $6728 \pm 1306$ & $6709 \pm 1544$ & 0.95 \\
\hline$\%$ of predicted REE (\%) & $100 \pm 15$ & $99 \pm 9$ & 0.69 \\
\hline \multicolumn{4}{|l|}{ Endocrine profile } \\
\hline Insulin (pmol/1) & $42 \pm 23$ & $39 \pm 19$ & 0.53 \\
\hline Leptin (ng/ml) & $9.5 \pm 7.7$ & $8.4 \pm 7.8$ & 0.60 \\
\hline Adiponectin $(\mu \mathrm{g} / \mathrm{ml})$ & $9.5 \pm 4.5$ & $14.0 \pm 6.5$ & $<0.01$ \\
\hline$\alpha-\mathrm{TNF}-\mathrm{R} 2(\mathrm{ng} / \mathrm{ml})$ & $1.38 \pm 0.48$ & $1.41 \pm 0.33$ & 0.81 \\
\hline \multicolumn{4}{|l|}{ Metabolic features } \\
\hline $\left.\operatorname{SI}_{\mathrm{P}(\text { clamp })}\left(1 \cdot \mathrm{min}^{-1} \cdot \mathrm{kg} \mathrm{LBM}^{-1} \cdot \mathrm{pmol}^{-1}\right)^{-1}\right) \times 10^{3}$ & $13.2 \pm 4.8$ & $17.8 \pm 6.0$ & $<0.01$ \\
\hline Lipid oxidation $\left(\mathrm{mg} \cdot \mathrm{kg} \mathrm{LBM}{ }^{-1} \cdot \mathrm{min}^{-1}\right)$ & $1.21 \pm 0.46$ & $1.25 \pm 0.37$ & 0.70 \\
\hline Soleus IMCL content (AU) & $88 \pm 48$ & $62 \pm 36$ & 0.03 \\
\hline Tibialis anterior IMCL content (AU) & $21 \pm 12$ & $15 \pm 10$ & 0.05 \\
\hline
\end{tabular}


Experimental protocol Subjects were instructed to consume a weight maintenance diet containing at least $250 \mathrm{~g}$ of carbohydrate per day and to abstain from exercise activity for 3 weeks before the studies. Women were studied between days 3 to 8 of the menstrual cycle. Subjects were studied by means of the euglycaemic-hyperinsulinaemic clamp and indirect calorimetry in order to assess whole-body insulin sensitivity, glucose and lipid oxidation (following correction for urinary nitrogen excretion) after a 10 -h overnight fast and during the insulin clamp. Within 2 to 3 days they were studied in the Division of Diagnostic Radiology of our institute (between 07.00 and 09.00 hours after a 10-h overnight fast) by means of ${ }^{1} \mathrm{H}-\mathrm{MRS}$ to measure IMCL content. Within 2 to 3 days they also underwent dual-energy $\mathrm{X}$-ray absorptiometry (DXA) to assess body composition.

Euglycaemic-hyperinsulinaemic clamp Subjects were admitted to the Metabolic Unit of the Division of Internal Medicine at 07.00 hours after a 10 -h overnight fast. A teflon catheter was inserted into an antecubital vein for infusions and an additional one was inserted retrogradely into a wrist vein for blood sampling. The hand was kept in a heated box $\left(50^{\circ} \mathrm{C}\right)$ to allow sampling of arterialised venous blood. A bolus (5 mg/kg of body weight) followed by a continuous infusion $(0.05 \mathrm{mg} / \mathrm{kg}$ of body weight per minute) of $6,6-{ }^{2} \mathrm{H}_{2}$ glucose obtained from massTrace (Woburn, MA, USA) was administered. Blood samples for postabsorptive plasma glucose, total cholesterol, triglycerides, NEFA, insulin, $\alpha$-tumour necrosis factor receptor-2 $(\alpha$-TNFR-2), leptin and adiponectin were performed in duplicate in the postabsorptive condition. Following a 150min tracer equilibration period, a euglycaemic-hyperinsulinaemic clamp was performed as previously described $[10,14]$ for a 150 -min period using an insulin infusion rate of $40 \mathrm{mU} \cdot \mathrm{m}^{-2} \cdot \mathrm{min}^{-1}$. Blood samples for plasma hormones, substrates and tracer enrichment were drawn every $15 \mathrm{~min}$ throughout the study.

Indirect calorimetry Indirect calorimetry was performed continuously, while subjects were lying quietly, for $30 \mathrm{~min}$ during the basal equilibration period and at the end of the clamp with a ventilated hood system (Sensor Medics 2900; Metabolic Measurement Cart, Milan, Italy). The mean CVs within the session for $\mathrm{O}_{2}$ and $\mathrm{CO}_{2}$ measurements were below $2 \%$.

${ }^{1} H$-magnetic resonance spectroscopy This was performed on a GE Signa 1.5 Tesla scanner (General Electric Medical Systems, Milwaukee, WI, USA) using a conventional linear extremity coil as previously described $[3,10,14]$. Briefly, two ${ }^{1} \mathrm{H}$ spectra were collected from a $15 \times 15 \times 15 \mathrm{~mm}$ volume within the soleus and tibialis anterior muscles respectively, using a PRESS pulse sequence $(\mathrm{TR}=2,000 \mathrm{~ms}$ and $\mathrm{TE}=60 \mathrm{~ms}$ ) and 128 averages were accumulated for each spectrum with a final acquisition time of $4.5 \mathrm{~min}$. The water signal was suppressed during the acquisition as it would dominate the other metabolites' peak signals of interest. A third ${ }^{1} \mathrm{H}$ spectrum of a triglyceride solution inside a glass sphere, positioned within the extremity coil next to the calf, was also obtained during the same session in order to have an external standard acquired in the same conditions as the subject's spectra. Postprocessing of the data, executed with Sage/IDL software (GE Medical Systems) was performed as previously described $[3,10,14]$. The T2 (spinspin relaxation time) value of the muscle $-\mathrm{CH}_{2}$ signal of the IMCL compartment was measured in five lean and four overweight individuals, in whom signal intensities were obtained at six different TEs chosen to linearly fit a monoexponential function, and was found to be not different between the subjects.

Body composition DXA was performed with a LunarDPX-IQ scanner (Lunar, Madison, WI, USA), as previously described $[3,10,14]$. Fat content is expressed as $\%$ of tissues.

Analytical procedures Plasma glucose was measured with a Beckman glucose analyser [3]. Plasma NEFA, total cholesterol, and triglycerides were measured as previously described [3]. Plasma insulin was measured using microparticle enzyme immunoassay technology as previously described $[10,14]$. Plasma leptin concentrations were determined as previously described $[10,14]$ by RIA with a human kit. $\alpha$-TNFR-2 was measured with an enzyme immunoassay following the manufacturer's (Immunotech; Beckman Coulter, Marseille, France) recommendations (intra- and interassay $\mathrm{CV}<3 \%$ and $5 \%$, respectively) as previously described $[10,14]$. Plasma adiponectin was measured using a commercially available radioimmunoassay kit (Linco Research, St Charles, MO, USA) as previously described [15]. The intra-assay and interassay CVs for adiponectin were $4 \pm 1 \%$ and $14 \pm 3 \%$, respectively. Serum urea nitrogen was measured in the postabsorptive and hyperinsulinaemic conditions using an enzymatic method on an Hitachi 747 [10]. The $\mathrm{d}_{2}$-glucose enrichment was measured by GCMS as previously described $[3,10,14,15]$.

Calculations Glucose turnover was calculated in the basal state by dividing the $6,6-{ }^{2} \mathrm{H}_{2}$ glucose infusion rate by the steady-state plateau of plasma $6,6-{ }^{2} \mathrm{H}_{2}$ glucose enrichment achieved during the last $45 \mathrm{~min}$ of the basal period. Glucose kinetics during the insulin clamp was calculated using Steele's equations for the non-steady state [16]. A steady state of plasma di-deuterated glucose enrichments was reached in the study groups during both the end of the equilibration period and the last $30 \mathrm{~min}$ of the insulin clamp. Endogenous glucose production (EGP) was calculated by subtracting the glucose infusion rate from the rate of glucose appearance measured with the isotope tracer technique. Total body glucose uptake was determined during the clamp by adding the rate of residual EGP to the exogenous glucose infusion rate. Insulin sensitivity $\left(\mathrm{SI}_{\mathrm{P} \text { (clamp) }}\right)$ was obtained as follows: $\Delta \mathrm{Rd} /(\Delta \mathrm{IxG})$, where $\Delta \mathrm{Rd}$ is the increment of total glucose uptake (normalised to $\mathrm{kg}$ lean body mass [LBM]), $\Delta I$ is the increment of plasma insulin concentration (both calculated at basal and clamp steady-state conditions), and $G$ is the plasma glucose concentration during the clamp [17]. Glucose, lipid and 
protein oxidation were estimated as previously described [10] in the postabsorptive state. During the insulin clamp, protein oxidation rates were corrected for changes in pool size [18].

Statistical analysis Data in text and tables are means \pm SD. Data in figures are means $\pm 95 \%$ CI. Analyses were performed using SSPS (version 10.0; SPSS, Chicago, IL, USA). The steady state for plasma glucose $6,6-{ }^{2} \mathrm{H}_{2}$ enrichment was defined as a non-significant correlation with time ( $p>0.05)$ using linear regression. An independent $t$-test was used to compare basal values between OFF and NOR for each variable. The effect of whole-body lipid oxidation was assessed after its segregation into quartiles within OFF and NOR. One-way ANOVA or Kruskal-Wallis non-parametric tests were used to compare variables between quartiles of lipid oxidation within OFF and NOR separately depending on the distribution of the data. Tukey's post hoc test was used. Regression analysis was performed using the General Linear Model procedure to detect significant effects of group-by-lipid oxidation interaction on $\mathrm{SI}_{\mathrm{P}(\text { clamp }) \text {, }}$ soleus and tibialis anterior IMCL content. Also in this case, non-parametric correlation coefficients were obtained using Spearman's rho when appropriate. Statistical significance was defined as a $p$ value of less than 0.05 .

\section{Results}

Anthropometric, laboratory and whole-body energetic features of study groups There was no difference between the study groups with regard to sex, age, BMI, body fat content and LBM (Table 1). The physical activity index also did not differ between the study groups (range 8.4-9.0 in OFF and 8.1-9.0 in NOR; Table 1). There was also no difference in fasting plasma glucose and triglyceride values between the groups. In contrast, OFF showed higher total cholesterol and fasting plasma NEFA (Table 1). Energy expenditure and the \% measured energy expenditure/ predicted energy expenditure (calculated using HarrisBenedict equations) ratio were not different between the groups.

Endocrine profile There was no difference in postabsorptive plasma insulin concentrations between the OFF and NOR groups (Table 1). During the clamp, the plasma insulin concentration reached a plateau, which also did not differ between the groups ( $380 \pm 79$ vs. $392 \pm 134 \mathrm{pmol} / \mathrm{l}$; $p=0.67$ ). Plasma leptin concentrations were also not different between groups (Table 1), while the plasma adiponectin concentration was lower in the OFF group than in the NOR group (Table 1). Plasma $\alpha$-TNFR-2 concentrations were not different between the groups (Table 1).

Insulin-stimulated glucose metabolism and insulin sensitivity The EGP was not different between the two groups, either in the fasting $\left(2.13 \pm 0.24 \mathrm{vs} .2 .23 \pm 0.32 \mathrm{mg} \cdot \mathrm{kg}^{-1} \cdot \mathrm{min}^{-1}\right.$; $p=0.29)$ or in the insulin-stimulated conditions $(0.33 \pm 0.18$ vs. $0.38 \pm 0.21 \mathrm{mg} \cdot \mathrm{kg}^{-1} \cdot \mathrm{min}^{-1} ; p=0.23$ ) (OFF and NOR groups, respectively). Whole-body insulin-stimulated glucose disposal was reduced in OFF $\left(6.60 \pm 2.09 \mathrm{mg} \cdot \mathrm{kg}^{-1} \mathrm{LBM}\right.$ $\left.\mathrm{min}^{-1}\right)$ in comparison with NOR $\left(8.77 \pm 1.97 \mathrm{mg} \cdot \mathrm{kg}^{-1} \mathrm{LBM}\right.$. $\left.\min ^{-1} ; p<0.001\right)$. This defect was due to the impairment of non-oxidative glucose metabolism (3.23 \pm 1.69 vs. $4.94 \pm 1.90$ $\mathrm{mg} \cdot \mathrm{kg}^{-1} \mathrm{LBM} \mathrm{min}^{-1}$ in OFF vs. NOR; $\left.p<0.001\right)$ since glucose oxidation $\left(3.52 \pm 1.61\right.$ vs. $3.83 \pm 1.89 \mathrm{mg} \cdot \mathrm{kg}^{-1} \mathrm{LBM} \mathrm{min}{ }^{-1}$; $p=0.42$ ) was not affected in OFF in comparison with NOR. $\mathrm{SI}_{\mathrm{P} \text { (clamp) }}$ was also reduced in OFF when compared with NOR (Table 1).

Lipid metabolism Fasting plasma NEFA were higher in OFF than in NOR (Table 1). During the insulin clamp, suppression of plasma NEFA was not different between groups ( $89 \pm 12$ vs. $92 \pm 9 \%$ in OFF and NOR, respectively; $p=0.19)$. Fasting lipid oxidation was not different in OFF when compared with NOR (Table 1). During the clamp lipid oxidation was suppressed by $46 \%\left(0.54 \pm 0.49 \mathrm{mg} \cdot \mathrm{kg}^{-1}\right.$ LBM $\left.\mathrm{min}^{-1}\right)$ in OFF and by $62 \%\left(0.45 \pm 0.31 \mathrm{mg} \cdot \mathrm{kg}^{-1}\right.$ LBM $\left.\cdot \min ^{-1}\right)$ in NOR $(p=0.19) .{ }^{1} \mathrm{H}-\mathrm{MRS}$ of the calf muscle showed that the IMCL content in the soleus and in the tibialis anterior muscles was higher in OFF than in NOR subjects (Table 1).

IMCL content and insulin sensitivity Our group previously reported a relationship between IMCL content and wholebody insulin sensitivity in non-obese individuals. In this work, in which non-obese and overweight/obese individuals were included, the preliminary search for significant interactions revealed the effect of a group-by-soleus IMCL interaction using $\mathrm{SI}_{\mathrm{P}(\mathrm{clamp})}$ as the dependent variable ( $p=$ 0.02 ). Then, we performed linear regression analysis in the entire study population $\left(r^{2}=0.21, p<0.0003\right)$ and separately in the lean individuals (body fat $<30 \% ; r^{2}=0.42, p<0.01$ ) and in the overweight individuals (body fat $>30 \% ; r^{2}=0.11$, $p=0.11$ ). The lack of association between soleus IMCL content and insulin sensitivity in the overweight/obese individuals was similar to a previous report by us on patients with type 2 diabetes [15] and patients with myotonic dystrophy type 1 [19].

Quartiles of lipid oxidation Individuals within the OFF group and the NOR group were subdivided into quartiles of fasting lipid oxidation. The quartile cutoffs were 1.041, 1.220 and $1.684 \mathrm{mg} \cdot \mathrm{kg}^{-1} \mathrm{LBM} \mathrm{min}^{-1}$ in OFF and 0.937 , 1.184 and $1.515 \mathrm{mg} \cdot \mathrm{kg}^{-1} \mathrm{LBM} \mathrm{min}^{-1}$ in NOR, respectively.

Effect of group and fasting whole-body lipid oxidation on insulin sensitivity $\mathrm{SI}_{\mathrm{P}(\mathrm{clamp})}$ was lower in OFF than in NOR (Fig. 1; general linear model analysis; $p<0.003)$. $\mathrm{SI}_{\mathrm{P}(\mathrm{clamp})}$ was different within quartiles of lipid oxidation in OFF $(p<0.05)$ but not within quartiles of lipid oxidation in NOR $(p=0.76)$ (Fig. 1). Lipid oxidation was significantly associated with $\mathrm{SI}_{\mathrm{P}(\text { clamp) }}$ in OFF (Spearman's rho 0.47; $p=$ 0.01 ) but not in NOR (Spearman's rho 0.13; $p=0.47$ ).

Effect of group and fasting whole-body lipid oxidation on IMCL content Soleus (Fig. 2) and tibialis anterior (Fig. 3) IMCL content was higher in OFF than in NOR (general 


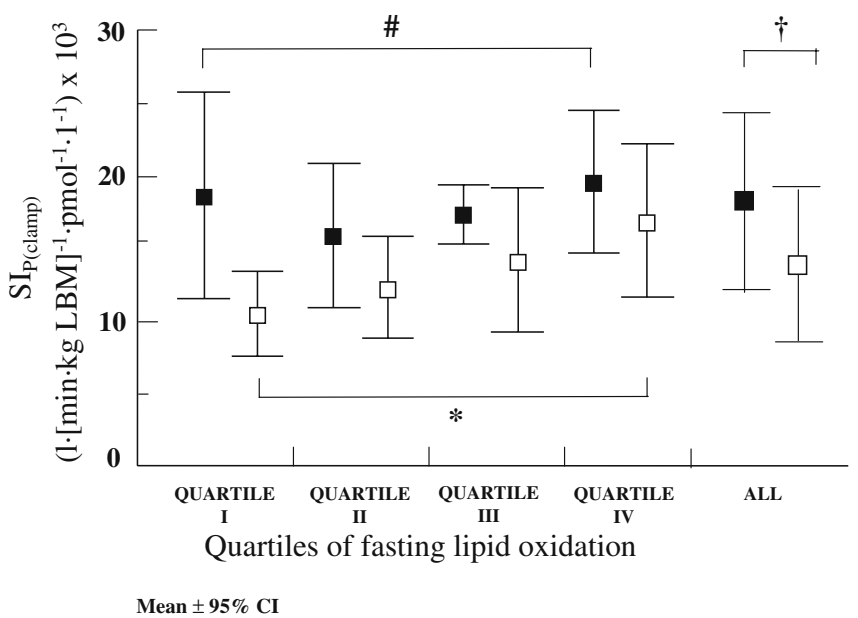

Fig. 1 Effect of group and fasting whole-body lipid oxidation on insulin sensitivity. Quartiles of fasting whole-body lipid oxidation are represented for OFF (open squares) and NOR (filled squares). Insulin sensitivity $\left(\mathrm{SI}_{\mathrm{P}(\text { clamp) }}\right)$ was lower in OFF than in NOR $\left({ }^{\dagger} p<\right.$ $0.003)$. $\mathrm{SI}_{\mathrm{P}(\text { clamp })}$ was different within quartiles of lipid oxidation in OFF $(* p<0.05)$ but not within quartiles of lipid oxidation in NOR $\left({ }^{\#} p=0.76\right)$. Moreover, a significant correlation between lipid oxidation and $\mathrm{SI}_{\mathrm{P}(\text { clamp) }}$ was found in OFF (Spearman's rho 0.47; $p=0.01$ ) but not in NOR (Spearman's rho $0.13 ; p=0.47$ ). Data are means \pm $95 \%$ CI

linear model analysis: $p<0.03$ and $p=0.05$, respectively). Soleus and tibialis anterior IMCL were not different within quartiles of lipid oxidation in OFF and NOR (Figs. 2, 3). No correlation between lipid oxidation and soleus or tibialis anterior IMCL content was found in OFF and NOR.

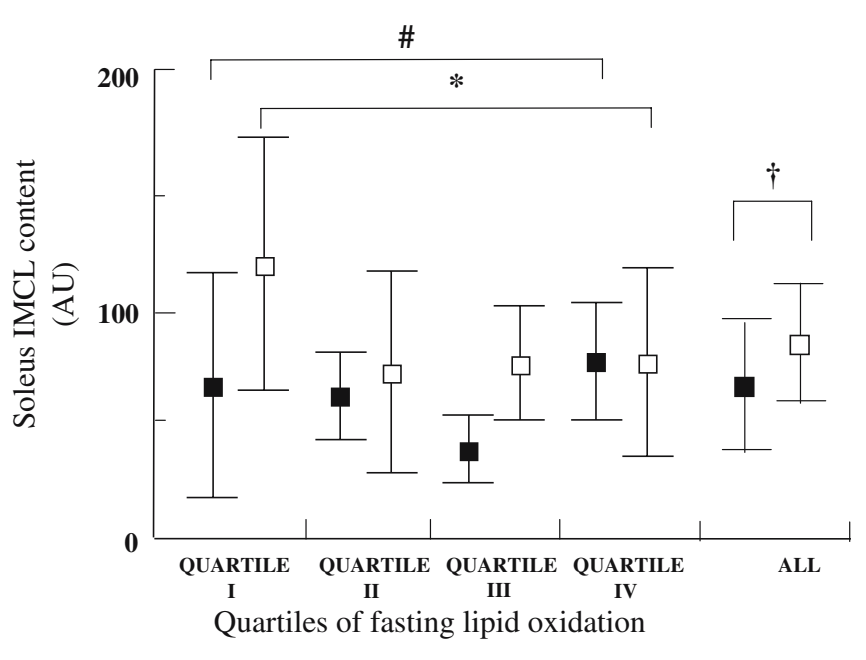

Mean $\pm 95 \%$ CI

Fig. 2 Effect of group and fasting whole-body lipid oxidation on soleus IMCL content. Quartiles of fasting whole-body lipid oxidation are represented for OFF (open squares) and NOR (filled squares). Soleus IMCL content was higher in OFF than in NOR $(p<0.03)$. Soleus IMCL was not different within quartiles of lipid oxidation in OFF $\left({ }^{*} p=0.35\right)$ and NOR $\left({ }^{*} p=0.36\right)$. No correlation between lipid oxidation and soleus IMCL content was found in OFF (Spearman's rho $-0.23 ; p=0.24$ ) and NOR (Spearman's rho 0.12; $p=0.50)$. Data are means $\pm 95 \%$ CI

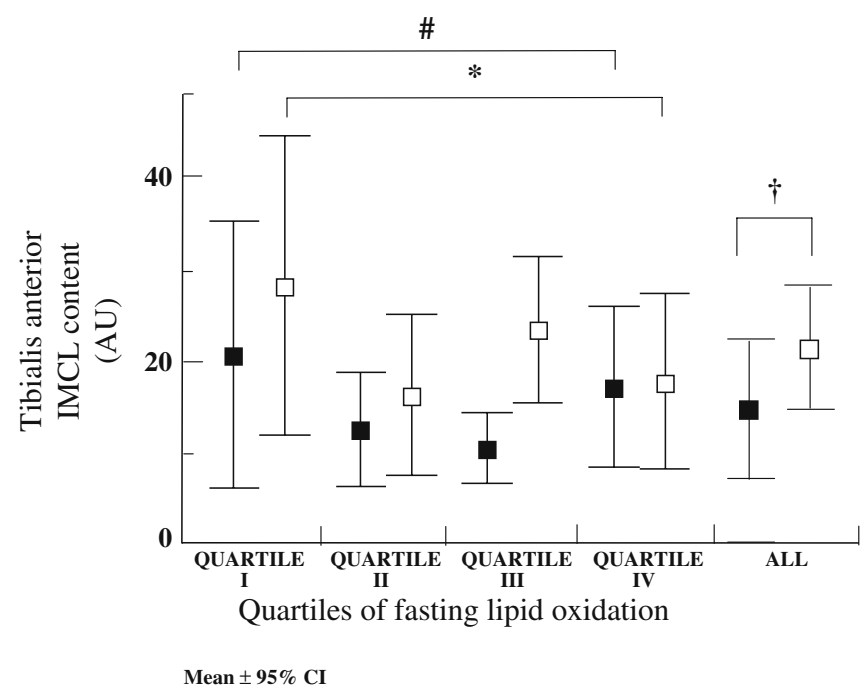

Fig. 3 Effect of group and fasting whole-body lipid oxidation on tibialis anterior IMCL content. Quartiles of fasting whole-body lipid oxidation are represented for OFF (open squares) and NOR (filled squares). Tibialis anterior IMCL content was higher in OFF than in NOR $\left({ }^{\top} p=0.05\right)$. Tibialis anterior IMCL was not different within quartiles of lipid oxidation in OFF $\left({ }^{*} p=0.32\right)$ and NOR $\left({ }^{*} p=0.28\right)$. No correlation between lipid oxidation and tibialis anterior IMCL content was found in OFF (Spearman's rho $-0.11 ; p=0.59$ ) and NOR (Spearman's rho $-0.12 ; p=0.53$ ). Data are means $\pm 95 \%$ CI

Effect of group and fasting whole-body lipid oxidation on plasma NEFA concentration Plasma NEFA concentrations were higher in OFF than in NOR (Table $1 ; p<0.01$ ). However, plasma NEFA concentrations were not different within quartiles of lipid oxidation in OFF $(0.577 \pm 0.205,0.709 \pm$ $0.219,0.665 \pm 0.200$ and $0.647 \pm 0.088 \mathrm{mmol} / \mathrm{l}$ in quartiles I, II, III and IV, respectively) and NOR $(0.410 \pm 0.182,0.385 \pm$ $0.172,0.576 \pm 0.212$ and $0.566 \pm 0.224 \mathrm{mmol} / 1$ in quartiles I, II, III and IV, respectively).

\section{Discussion}

This work confirmed that in offspring of type 2 diabetic parents with an established increased risk of developing type 2 diabetes [20], peripheral insulin resistance is associated with higher circulating NEFA and IMCL content. The novel finding is that insulin sensitivity improved across increasing quartiles of fasting lipid oxidation within the OFF group but remained constant in the NOR group, suggesting that impaired fat oxidation may be a primary pathogenic factor of insulin resistance in people with a genetic background for type 2 diabetes.

A 30 to $40 \%$ lower insulin sensitivity in offspring of type 2 diabetic parents is a typical feature [2, 3], even if only $25 \%$ of these individuals actually had severe insulin resistance [21]. We confirmed that in these subjects the severity of the impairment of insulin-stimulated glucose metabolism was proportional with higher IMCL content and fasting plasma NEFA concentrations (Table 1). Hypothesising that these abnormalities of lipid metabolism might be due to altered lipid oxidation, we performed whole-body 
indirect calorimetry to search for a relationship between whole-body lipid oxidation and (1) insulin sensitivity, and (2) IMCL content. At first glance, fasting lipid oxidation was not different in the two groups (Table 1) and was similarly suppressed ( $46 \pm 32 \%$ vs. $62 \pm 27 \%$; $p=0.58$ ) during the insulin clamp. This finding was in apparent contrast with a recent study [22], which used magnetisation transfer experiments to demonstrate, in a cohort of OFF previously known to be insulin-resistant, that abnormal IMCL content was associated with a skeletal muscle defect in mitochondrial oxidative phosphorylation and a reduced ATP synthesis rate. This discrepancy was reconciled using a similar selection procedure for study subjects; the analysis was performed based on segregation of our OFF group by quartiles of lipid oxidation. As shown in Fig. 1, this analysis demonstrated that the OFF subgroup with the lowest fasting whole-body lipid oxidation (quartile I) was that with the most severe insulin-resistant state, while the OFF subgroup with the highest fasting lipid oxidation rates was that with the highest insulin sensitivity. Consensual results were obtained when analysis was performed segregating the OFF group by quartiles of $\mathrm{SI}_{\mathrm{p} \text { (clamp) }}$ (the most insulin-resistant being the individuals with the lowest fasting whole-body lipid oxidation; data not shown). Moreover a significant association between insulin sensitivity and fasting wholebody lipid oxidation was demonstrated, supporting the original hypothesis that an impairment of fat oxidation may be associated with insulin resistance. This conclusion was further sustained by the observation that the relationship linking fasting whole-body lipid oxidation and insulin sensitivity was not detectable in the NOR control group, when it was segregated by quartiles of lipid oxidation, suggesting that this alteration may be peculiar to individuals with a genetic risk of developing type 2 diabetes in the future. A disturbed oxidative enzyme activity has also been described in the skeletal muscle of type 2 diabetic and obese individuals using an in vitro studies approach $[23,24]$. A dysfunction of mitochondria was proposed as a possible cause of the impairment of lipid oxidation in the skeletal muscle of type 2 diabetic patients [25], and a specific age-associated decline in mitochondrial function has been suggested as a possible pathogenic factor in the development of insulin resistance in the elderly [26]. A primary mitochondrial dysfunction as a cause of IMCL accumulation may constitute a self-perpetuating mechanism inducing a damage to mitochondria via ROS production [27]. It has been suggested that the molecular mechanisms behind the abnormal pattern of lipid oxidation may be linked with a common polymorphism of the PPAR- $\gamma$ coactivator 1 (a transcriptional regulator of genes responsible for mitochondrial biogenesis and fat oxidation) in a Danish population [28] and in Pima Indians [29]. In addition, in the skeletal muscle of patients with type 2 diabetes, there was a reduction in the expression of genes encoding the PPAR $-\gamma$ coactivator 1 $[30,31]$.

The second aim of this work was to test whether the impairment of whole-body lipid oxidation was also associated with abnormal IMCL accumulation. Originally IMCL content was described as strongly associated with insulin resistance [1-3] in sedentary and lean individuals. Additional studies revealed that the IMCL content is also strongly influenced by the aerobic fitness status of the study groups and by body composition [32]. In particular, the association between IMCL content and insulin sensitivity found in the lean individuals was lost when overweight/ obese individuals were studied [33]. We also confirmed a lack of association in the individuals with body fat greater than $30 \%$. Moreover, we observed that in contrast to insulin sensitivity, the soleus (Fig. 2) and tibialis anterior (Fig. 3) IMCL content in both the OFF and NOR groups remained constant across increasing quartiles of fasting lipid oxidation, suggesting that the impact of lipid oxidation on insulin sensitivity is not directly mediated via an effect on IMCL content but is rather complex and mediated by other factors.

Since studies have also implicated several adipocytederived hormones in the cause of insulin resistance modulating IMCL content and possibly fat oxidative disposal, we measured plasma concentrations of leptin, adiponectin and $\alpha$-TNF-R-2 in the fasting condition (Table 1). Adiponectin concentrations were significantly lower in the OFF than in the NOR group, and a weak correlation with $\mathrm{SI}_{\mathrm{p} \text { (clamp) }}$ was found when data of study subjects were plotted all together $\left(r^{2}=0.07 ; p=0.04\right)$, but not when plotted separately between OFF or NOR; in fact, a group-by-adiponectin effect on $\mathrm{SI}_{\mathrm{p}(\mathrm{clamp})}$ was found Moreover, no association was found between adiponectin and IMCL content or lipid oxidation when tested with both parametric and nonparametric tests. Our data suggested that alterations in plasma concentrations of these adipocyte-derived factors do not have a major role in mediating insulin resistance, lipid oxidation and IMCL content, and that the role of the association of adiponectin with $\mathrm{SI}_{\mathrm{p} \text { (clamp) }}$ remains to be clarified in terms of biological relevance.

A technical limitation of our study is that the level of physical activity was not determined using a direct parameter but was estimated using a questionnaire; due to the criteria of selection and to the consequent homogeneity of the features of the study subjects and due to the lack of statistical association, we can rule out that the observed differences in lipid oxidation between and within groups may be primarily due to overall or specific types of exercise. An additional limitation of the study is that lipid oxidation was assessed using indirect calorimetry reflecting substrate oxidation of all tissues and not only of the skeletal muscle; we believe that this limitation was partially circumvented by correcting the lipid oxidation for the LBM. Nevertheless we cannot exclude that the association between lipid oxidation and insulin sensitivity would have been stronger if more specific measures of skeletal muscle lipid oxidation had been employed.

In conclusion, the present work confirmed the higher IMCL content in the OFF group and its relationship with $\mathrm{SI}_{\mathrm{p} \text { (clamp) }}$, but failed to demonstrate an association between fasting lipid oxidation and IMCL content within groups. Despite that, it was demonstrated that insulin sensitivity improved across increasing quartiles of fasting lipid oxidation within the OFF group and remained constant in the NOR group, supporting the hypothesis that impaired fat 
oxidation may be a primary pathogenic factor of insulin resistance in people with a genetic background for type 2 diabetes.

Acknowledgements We wish to thank Antonella Scollo (Registered Nurse) of the Metabolic Unit of the Istituto Scientifico H San Raffaele for nursing assistance. This work was supported by grants from the Italian Minister of Health (RF99.55, RF01.1831).

\section{References}

1. Krssak M, Falk Petersen K, Dresner A et al (1999) Intramyocellular lipid concentrations are correlated with insulin sensitivity in humans: a ${ }^{1} \mathrm{H}$ NMR spectroscopy study. Diabetologia 42:113-116

2. Jacob S, Machann J, Rett K et al (1999) Association of increased intramyocellular lipid content with insulin resistance in lean nondiabetic offspring of type 2 diabetic subjects. Diabetes 48:1113-1119

3. Perseghin G, Scifo P, De Cobelli F et al (1999) Intramyocellular triglyceride content is a determinant of in vivo insulin resistance in humans: a ${ }^{1} \mathrm{H}-{ }^{13} \mathrm{C}$ NMR spectroscopy assessment in offspring of type 2 diabetic parents. Diabetes 48:1600-1606

4. Coldberg SR, Simoneau JA, Thaete FL, Kelley DE (1995) Skeletal muscle utilization of free fatty acids in women with visceral obesity. J Clin Invest 95:1846-1853

5. Kelley DE, Goodpaster B, Wing RR, Simoneau JA (1999) Skeletal muscle fatty acid metabolism in association with insulin resistance, obesity and weight loss. Am J Physiol 277: E1130-E1141

6. Blaak EE, Wagenmakers AJM, Glatz JFC et al (2000) Plasma FFA utilization and fatty acid-binding protein content are diminished in type 2 diabetic muscle. Am J Physiol Endocrinol Metab 279:E146-E154

7. Blaak EE, Wolffenbuttel BH, Saris WH, Pelsers MM, Wagenmakers AJ (2001) Weight reduction and the impaired plasma-derived free fatty acid oxidation in type 2 diabetic subjects. J Clin Endocrinol Metab 86:1638-1644

8. Mensink M, Blaak EE, van Baak MA, Wagenmakers AJ, Saris WH (2001) Plasma free fatty acid uptake and oxidation are already diminished in subjects at high risk for developing type 2 diabetes. Diabetes 50:2548-2554

9. Luzi L, Perseghin G, Tambussi G et al (2003) Intramyocellular lipid accumulation and reduced whole body lipid oxidation in HIV infected patients with lipodystrophy. Am J Physiol Endocrinol Metab 284:E274-E280

10. Perseghin G, Scifo P, Danna M et al (2002) Normal insulin sensitivity and IMCL content in overweight humans are associated with higher fasting lipid oxidation. Am J Physiol Endocrinol Metab 283:E556-E564

11. Goodpaster BH, Katsiaras A, Kelley DE (2003) Enhanced fat oxidation through physical activity is associated with improvements in insulin sensitivity in obesity. Diabetes 52:2191-2197

12. Gan SK, Kriketos AD, Ellis BA, Thompson CH, Kraegen EW, Chisholm DJ (2003) Changes in aerobic capacity and visceral fat but not myocyte lipid levels predict increased insulin action after exercise in overweight and obese men. Diabetes Care 26:1706-1713

13. Baecke JAH, Burema J, Frijters JER (1982) A short questionnaire for the measurement of habitual physical activity in epidemiological studies. Am J Clin Nutr 36:936-942

14. Perseghin G, Scifo P, Pagliato E et al (2001) Gender factors affect fatty acid-induced insulin resistance in nonobese humans: effects of oral steroidal contraception. J Clin Endocrinol Metab $86: 3188-3196$
15. Perseghin G, Lattuada G, Danna M et al (2003) Insulin resistance, intramyocellular lipid content and plasma adiponectin concentrations in patients with type 1 diabetes. Am J Physiol Endocrinol Metab 285:E1174-E1181

16. Steele R (1959) Influence of glucose loading and of injected insulin on hepatic glucose output. Ann NY Acad Sci 82:420 431

17. Bergman RN, Finegood DT, Ader M (1985) Assessment of insulin sensitivity in vivo. Endocr Rev 6:45-86

18. Tappy L, Owen OE, Boden G (1988) Effect of hyperinsulinemia on urea pool size and substrate oxidation rates. Diabetes 37 : $1212-1216$

19. Perseghin G, Comola M, Scifo P et al (2004) Postabsorptive and insulin-stimulated energy and protein metabolism in patients with myotonic dystrophy type 1 . Am J Clin Nutr 80:357364

20. Warram JH, Martin BC, Krolewski AS, Soeldner JS, Kahn CR (1990) Slow glucose removal rate and hyperinsulinemia precede the development of type II diabetes in the offspring of diabetic parents. Ann Int Med 113:909-915

21. Perseghin G, Ghosh S, Gerow K, Shulman GI (1997) Metabolic defects in lean, non-diabetic offspring of NIDDM parents: a cross-sectional study. Diabetes 46:1001-1009

22. Petersen KF, Dufour S, Befroy D, Garcia R, Shulman GI (2004) Impaired mitochondrial activity in the insulin-resistant offspring of patients with type 2 diabetes. N Engl J Med 350:664671

23. He J, Watkins S, Kelley DE (2001) Skeletal muscle lipid content and oxidative enzyme activity in relation to muscle fiber type in type 2 diabetes and obesity. Diabetes 50:817-823

24. Gaster M, Rustan AC, Aas V, Beck-Nielsen H (2004) Reduced lipid oxidation in skeletal muscle from type 2 diabetic subjects may be of genetic origin. Evidence from cultured myotubes. Diabetes 53:542-548

25. Kelley DE, He J, Menshikova EV, Ritov VB (2002) Dysfunction of mitochondria in human skeletal muscle in type 2 diabetes. Diabetes 51:2944-2950

26. Petersen KF, Befroy D, Dufour S et al (2003) Mitochondrial dysfunction in the elderly: possible role in insulin resistance. Science 300:1140-1142

27. Schrauwen P, Hesselink MKC (2004) Oxidative capacity, lipotoxicity, and mitochondrial damage in type 2 diabetes. Diabetes 53:1412-1417

28. Ek J, Andersen G, Urhammer SA et al (2001) Mutation analysis of peroxisome proliferator-activated receptor-gamma coactivator-1 (PGC-1) and relationships of identified amino acid polymorphisms to type II diabetes mellitus. Diabetologia 44: 2220-2226

29. Muller YL, Bogardus C, Beamer BA, Shuldiner AR, Baier LJ (2003) A functional variant in the peroxisome proliferator-activated receptor gamma2 promoter is associated with predictors of obesity and type 2 diabetes in Pima Indians. Diabetes 52: $1864-1871$

30. Mootha VK, Lindgren CM, Eriksson KF et al (2003) PGClalpha-responsive genes involved in oxidative phosphorylation are coordinately downregulated in human diabetes. Nat Genet 34:267-273

31. Patti ME, Butte AJ, Crunkhorn S et al (2003) Coordinated reduction of genes of oxidative metabolism in humans with insulin resistance and diabetes: potential role of PGC1 and NRF1. Proc Nat Acad Sci U S A 100:8466-8471

32. Thamer C, Machann J, Bachmann O et al (2003) Intramyocellular lipids: anthropometric determinants and relationships with maximal aerobic capacity and insulin sensitivity. J Clin Endocrinol Metab 88:1785-1791

33. Gan SK, Kriketos AD, Poynten AM et al (2003) Insulin action, regional fat, and myocite lipid: altered relationships with increased adiposity. Obes Res 11:1295-1305 